\title{
Giant enhanced g-factors in an InSb two-dimensional gas
}

\author{
B. Nedniyom, ${ }^{1}$ R. J. Nicholas, ${ }^{1, *}$ M. T. Emeny, ${ }^{2}$ L. Buckle, ${ }^{2}$ A. M. Gilbertson, ${ }^{2}$ P. D. Buckle, ${ }^{2}$ and T. Ashley ${ }^{2}$ \\ ${ }^{1}$ Department of Physics, Oxford University, Clarendon Laboratory, Parks Rd., Oxford OX1 3PU, United Kingdom \\ ${ }^{2}$ QinetiQ, Malvern Technology Centre, St. Andrews Rd., Malvern WR14 3PS, United Kingdom
}

(Received 12 November 2008; revised manuscript received 28 July 2009; published 24 September 2009)

\begin{abstract}
We report measurements of the electron g-factor in InSb quantum wells using the coincidence technique, polarization transition, and temperature-dependent resistivity. All three methods show that there is a giant enhancement of the spin slitting which is proportional to the spin polarization. Electron Zeeman energies as high as $51 \mathrm{meV}$ are measured leading to the conclusion that the additional contribution to the spin splitting is of order $30 \mathrm{meV}$, more than ten times larger than expected from conventional theories.
\end{abstract}

DOI: $10.1103 /$ PhysRevB.80.125328

PACS number(s): 73.43.Qt, 73.21.Fg, 75.30.Et

The influence of Coulomb interactions on the spin susceptibility and effective mass in two-dimensional electron and hole systems has been the subject of intense interest and speculation recently ${ }^{1-3}$ with measurements suggesting a significant enhancement of the spin susceptibility at very lowdensities materials with small g-factors such as GaAs-based systems, ${ }^{4-7} \mathrm{CdTe}^{8}$ and silicon. ${ }^{9,10}$ Such an effect is a key signature of many-body effects and has been studied theoretically for many years, however, to date only a few of the experimental analysis techniques have considered the influence of the changing spin polarization on the observed properties, ${ }^{6,8}$ as pointed out theoretically. ${ }^{1}$ Traditionally this phenomenon has been known as exchange enhancement of the g-factor ${ }^{11}$ with the origin of the enhancement attributed to the spin polarization of individual Landau levels. In addition to collective effects, single-particle spin splitting is also known to be enhanced by the presence of structural inversion asymmetry $^{12}$ (known as the Rashba effect) in which spinorbit coupling causes a zero magnetic field spin splitting. This second phenomenon is known to be particularly strong in narrow-gap semiconductors. ${ }^{13}$ Here we examine these phenomena in the narrow-gap semiconductor, InSb, where we demonstrate a strong systematic dependence of the electron g-factor on the electronic polarization and show that this collective effect is dominant at high fields and shows the predicted dependence upon total spin polarization. The magnitude of the g-factor enhancement is considerably larger than expected from the theory suggesting that the mechanism is not fully understood but the resulting giant effective $\mathrm{g}$-factors may have very significant implications for applications in spintronics.

Previous investigations of Zeeman splitting in narrowergap semiconductors have usually led to indications of g-factor enhancement ${ }^{14-18}$ although some authors suggest that the effect is small. ${ }^{19}$ To date however no systematic studies comparable to the work performed in GaAs and silicon have been reported. One difficulty is the influence of band nonparabolity which significantly increases the effective mass and decreases the g-factor relative to the bulk materials. This is also combined with significantly lower mobilities in these materials, particularly for low-density samples.

The two methods most commonly used to study the g-factor are known as the coincidence method ${ }^{20,21}$ which uses tilted magnetic fields to cause coincidences between spin-split levels with different Landau index and the observation of the polarization transition ${ }^{5,6,9,10}$ in parallel magnetic field at the condition $g^{*} \mu_{B} B=2 E_{F}$, where the Zeeman splitting is sufficiently large to completely polarize the electron system. The Zeeman splitting can also be analyzed using the high temperature dependence of the resistivity ${ }^{22}$ using the well-known Lifshitz-Kosevich (LK) formula ${ }^{23}$

$$
\Delta \rho_{x x} \propto \frac{X}{\sinh X} \exp \left(-\frac{\pi}{\omega_{c} \tau_{s}}\right) \cos [\pi(\nu+1 / 2)],
$$

where the factor $X=2 \pi^{2} k T / E_{g}$ and $E_{g}$ is the energy gap corresponding to either the cyclotron or Zeeman energy as appropriate. Here we use all three methods which are shown to give similar results under equivalent conditions.

The samples studied consist of $\mathrm{InSb} / \mathrm{Al}_{x} \mathrm{In}_{1-x} \mathrm{Sb}$ heterostructures grown on a semi-insulating GaAs substrate by molecular beam epitaxy. ${ }^{24}$ The carriers are confined within 15 , 20 , or $30 \mathrm{~nm} \mathrm{InSb}$ quantum wells, with asymmetric doping and barriers with $10 \%$ and $15 \%$ Aluminum content. The samples were fabricated into conventional Hall bars with typical carrier concentrations of $2-7 \times 10^{15} \mathrm{~m}^{-2}$ and mobilities of $2-15 \mathrm{~m}^{2} \mathrm{~V}^{-1} \mathrm{~s}^{-1}$ as given in Table I. Magnetotransport measurements were performed between $25 \mathrm{mK}$ and 100 $\mathrm{K}$ using superconducting magnets and a dilution refrigerator and conventional Helium variable-temperature cryostat. Typical measurement currents were $20 \mathrm{nA}$ in Hall bars of width $75 \mu \mathrm{m}$. Both measurement inserts were equipped with rotation mechanisms which could rotate the samples relative to the magnetic field direction in situ. Similar lowtemperature results were found on rotation at both $25 \mathrm{mK}$ and $1.5 \mathrm{~K}$ and all data shown in the figures were taken at 1.5

TABLE I. Table showing the sample parameters for the four main samples studied.

\begin{tabular}{lccc}
\hline \hline Sample & $\begin{array}{c}\text { Well width } \\
(\mathrm{nm})\end{array}$ & $\begin{array}{c}\text { Carrier density } \\
\left(\times 10^{15} \mathrm{~m}^{-2}\right)\end{array}$ & $\begin{array}{c}\text { mobility } \\
\left(\mathrm{m}^{2} \mathrm{~V}^{-1} \mathrm{~s}^{-1}\right)\end{array}$ \\
\hline W1 & 30 & $1.9-2.3$ & 11 \\
M1 & 20 & $2.7-3.8$ & 2 \\
N1 & 15 & $2.6-3.3$ & 15 \\
$\mathrm{~N} 2$ & 15 & 7.0 & 9 \\
\hline \hline
\end{tabular}




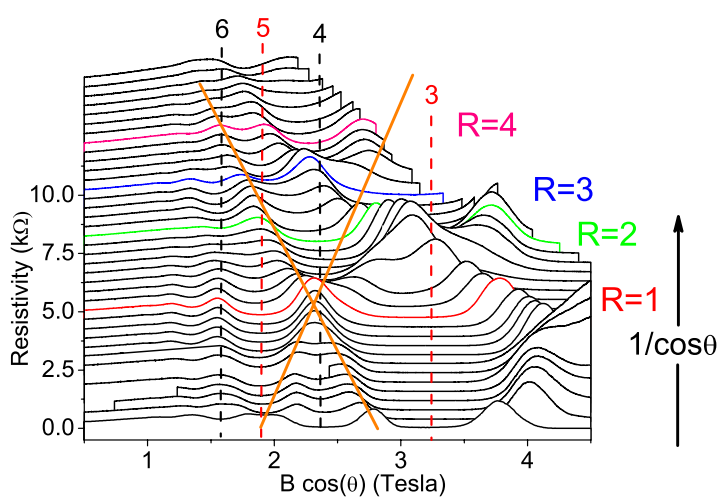

FIG. 1. (Color online) A series of magnetoresistivity measurements for sample W1 plotted as a function of the perpendicular component of magnetic field $(\mathrm{B} \cos \theta)$ for progressively increasing angles. Inset: a schematic of Landau levels for a fixed $\mathrm{B} \cos \theta$.

$\mathrm{K}$ as this enabled a greater number of angles to be studied.

A typical series of magnetoresistance measurements at different rotation angles are shown in Fig. 1 as a function of the perpendicular component of magnetic field. The perpendicular component of magnetic field was determined by measuring the angle from the slope of the low-field Hall coefficient. This method is estimated to give an accuracy in $\cos \theta$ of $\pm 2 \%$. The data clearly demonstrate the initial enhancement of the relative strength of the spin-split minima at odd values of the level occupancy $\nu$ due to the dependence of the spin splitting upon total magnetic field, compared with the Landau splitting which is proportional to the perpendicular component. The schematic dependence of the levels upon tilting is shown in the inset, which demonstrates that coincidences will occur between the two sets of levels at the condition

$$
R \cos \theta=\frac{g^{*} m^{*} \mu_{B}}{\hbar e},
$$

where the index, $R$, denotes the order of the coincidence and corresponds to the number of levels which have crossed over. The coincidence causes the merging of the splitresistivity peaks which occurs alternately for even and odd values of $\nu$ at odd and even values of $R$. The only exception to this is the $\nu=2$ crossing where the Ising quantum-Hall ferromagnet state is formed causing a resistance spike at the crossing, ${ }^{25}$ as reported previously. ${ }^{16,26}$ An accurate measurement of the coincidence is made by plotting the resistivity at integer occupancies which shows a peak as a function of angle, as shown in Fig. 2. Similarly we expect condition 2 to lead to a maximum spin gap when $R$ and $\nu$ are either both even or both odd and hence produce a minimum in the resistivity.

In order to deduce the g-factors from Eq. (2) it is necessary to know the electron effective mass. This was measured by fitting the temperature dependence of the oscillation amplitudes to the LK formula (Eq. (1)) for all of the samples studied for several different cool-down sequences (which generated slightly different electron densities). The results are shown in Fig. 3 and are found to agree well with predic-

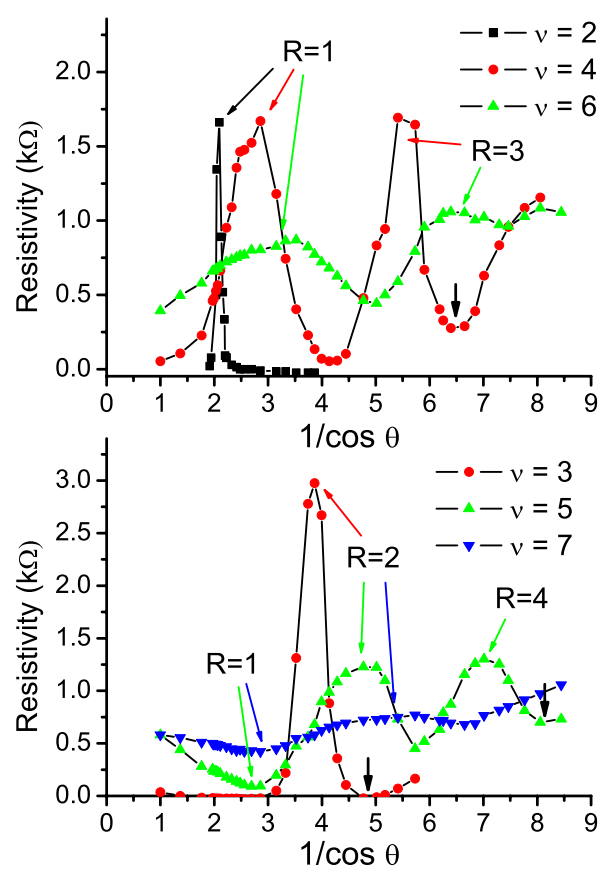

FIG. 2. (Color online) Plots of the resistivity values as a function of $1 / \cos \theta$ at the integer occupancy values $\nu=2-7$. The larger downward arrows indicate the point where the system becomes fully spin polarized for $R=3,4$, and 5 at $\nu=3,4$, and 5 .

tions from simple four-band $\mathbf{k} \cdot \mathbf{p}$ theory. The effective-mass values are typically in the range $0.018-0.026 m_{e}$, depending on the well width, carrier density, and hence Fermi level.

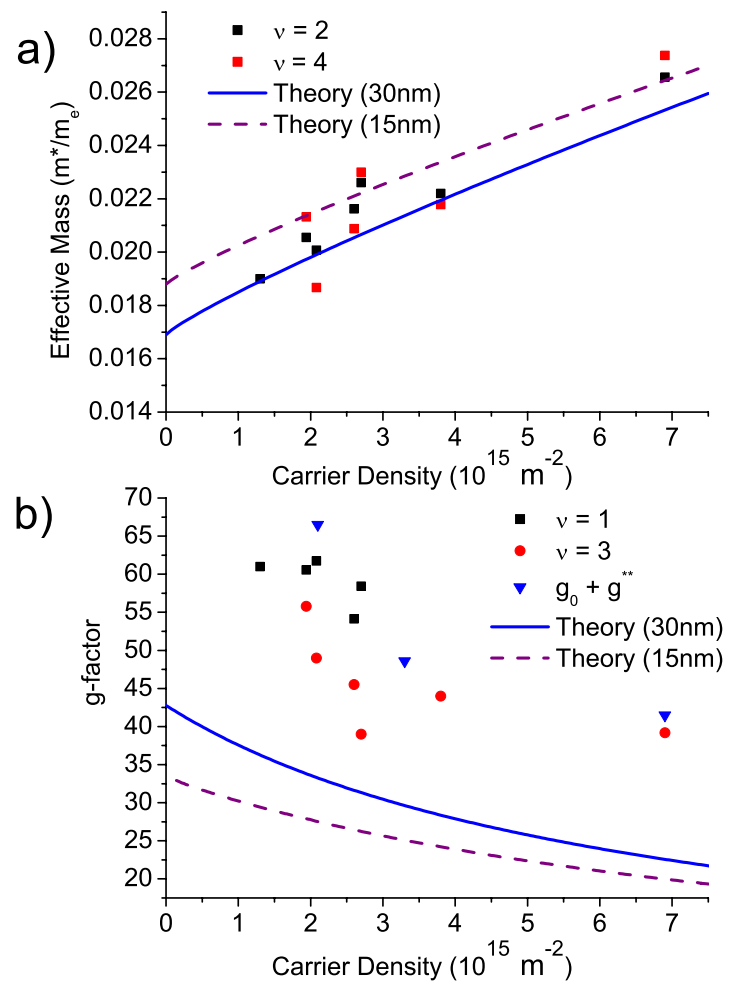

FIG. 3. (Color online) Experimental and theoretical dependencies of (a) the effective mass and (b) the g-factor as a function of density. 

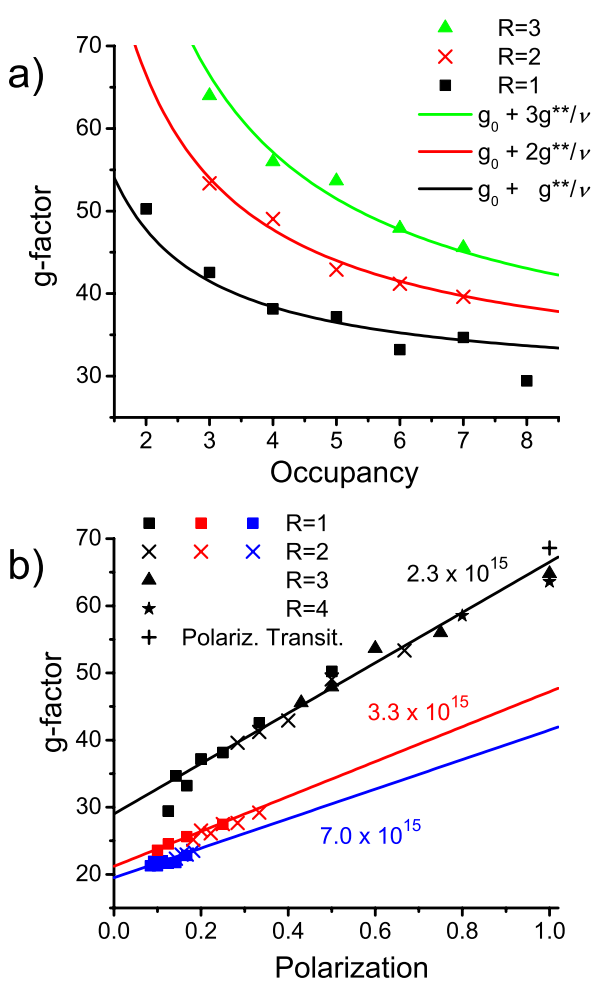

FIG. 4. (Color online) Experimentally determined g-factors as a function of (a) occupancy, $\nu$ for sample $\mathrm{W} 1$ and (b) total spin polarization $\xi=\frac{R}{\nu}$ for samples $\mathrm{W} 1, \mathrm{~N} 1$, and $\mathrm{N} 2$.

These values agree well with cyclotron resonance values reported for $30 \mathrm{~nm} \mathrm{InSb/InAlSb}$ quantum wells by both ourselves $^{27}$ and other authors ${ }^{28}$ but are substantially larger than the bulk InSb band-edge value of $0.0138 m_{e}$ (Ref. 29) due to the effects of nonparabolicity.

Using the effective-mass values we calculate the g-factors for different values of $\nu$ and $R$. An example of these is shown in Fig. 4(a) for the 30-nm-width sample, W1. There are two immediate conclusions from this, first that the g-factor increases when the occupancy, $\nu$, falls and second that the values for the g-factor increase with the coincidence index, $R$. Similar behavior is seen for all of the samples studied. It is clear from Fig. 4 that there is a well-defined and systematic dependence of the g-factor upon $\nu$ and $R$. The origin of this behavior becomes clear when we replot the g-factors in terms of the spin polarization of the total system $\xi$ which is given by

$$
\xi=\frac{n \uparrow-n \downarrow}{n \uparrow+n \downarrow}=\frac{R}{\nu}
$$

at the coincidence conditions. Plotting the data for three samples for all coincidences as a function of $\xi$ alone in Fig. 4(b) shows a remarkably consistent set of results. This demonstrates that the electron g-factor can be described in terms of two components, $g_{0}$, a constant polarization independent term and $g^{* *}$ which gives the proportionality to polarization, giving

$$
g^{*}=g_{0}+g^{* *} \times \xi
$$

TABLE II. Table showing the values of $g_{0}$ and $g^{* *}$ as deduced from the resistivity measurements, compared with the values of $\mathrm{m}^{*} / \mathrm{m}_{e}$ and $g_{E_{F}}$ calculated from conventional $\mathbf{k}$. p theory.

\begin{tabular}{lccccl}
\hline \hline Sample & $\begin{array}{c}\text { Well width } \\
(\mathrm{nm})\end{array}$ & $m^{*} / m_{e}$ & $g_{E_{F}}$ & $g_{0}$ & $g^{* *}$ \\
\hline $\mathrm{W} 1$ & 30 & 0.0202 & 32.6 & 29 & 37.5 \\
$\mathrm{~N} 1$ & 15 & 0.0228 & 24.9 & 21.2 & 26 \\
$\mathrm{~N} 2$ & 15 & 0.0266 & 19.9 & 19.5 & 22 \\
\hline \hline
\end{tabular}

The values deduced for $g_{0}$ and $g^{* *}$ are given in Table II together with the values expected for the single-particle g-factor using the same k.p calculations which correctly describe the effective mass and which are also shown in Fig. 3. It can be seen that the values for $g_{0}$ are quite close to the predicted single-particle values, consistent with the fact that the value deduced for an unpolarized gas is essentially the "bare" g-factor unenhanced by electron-electron interactions which are expected to be weak in this system. The dependence of the g-factor on polarization is qualitatively consistent with the predictions of Zhang and Das Sarma ${ }^{1}$ who have shown that the spin susceptibility should be strongly polarization dependent but the magnitude of the effect is much larger than their predictions. Experimental evidence for a polarization dependence of the g-factor was found by Zhu et al. ${ }^{6}$ for GaAs at much lower densities. These authors reported an empirical formula which predicts strong polarization-dependent effects at higher densities but were reluctant to extrapolate their results too far due to the increasing presence of discrepancies in describing the polarization transition. In previous reports of "exchange enhancement" of the g-factor it has usually been assumed that the enhancement was only produced by a population difference in any single Landau level. ${ }^{30}$ The largest values for the g-factor were usually found at occupancies where the resistivity has a strong minimum corresponding to a fully polarized Landau level but also to a higher average polarization as the strongest minima corresponded to lower occupancies (Eq. (3)). Careful inspection of Fig. 4 shows very slightly larger values for $g^{*}$ when resistivity minima ( $\nu$ odd, $R=1$ and $\nu$ even, $R=2$ ) are used to deduce the coincidence condition 2 compared to the results from maxima, where the system consists of two partially polarized levels, but the differences are small.

Even for measurements made on fully polarized single Landau levels where there is a pronounced resistivity minimum at low temperatures, the g-factors for the InSb system are still found to be dependent upon the total polarization. Figure 3 shows values of the g-factor deduced from the temperature dependence of the resistivity using the LK formula for the strongest minima with $\nu=1,3$ and $\theta=0$. At $\nu=1$ there is good agreement between the values deduced from the excitation gap and the projected value, $\left(g_{0}+g^{* *}\right)$, at $\xi=1$, deduced from the coincidence method. For $\nu=3$ we observe significantly smaller values for the g-factor which are much closer to the calculated single-particle values, despite the fact that at low temperature the minima are completely resolved and at least as far as the extended states are concerned, ${ }^{31}$ the 


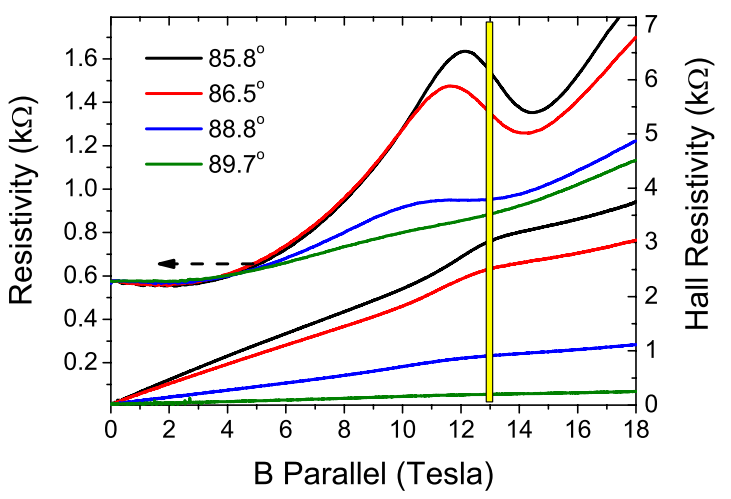

FIG. 5. (Color online) Magnetoresistance and Hall voltage for sample $\mathrm{W} 1$ at angles close to $90^{\circ}$ as a function of total field. The polarization transition takes place at $13 \mathrm{~T}$, causing a decrease in the resistivity and an anomaly in the Hall voltage.

individual Landau level is completely spin polarized.

Further confirmation of the enhanced g-factors deduced here comes from observing the polarization transition ${ }^{5,6,9,10}$ which takes place for fields close to the parallel orientation. This is shown in Fig. 5 where a significant magnetoresistance is seen which becomes larger as the quantum well is rotated away from exact parallel orientation, thus producing a small perpendicular component of magnetic field. This suggests that the origin of the magnetoresistance is due to elastic spin-orbit scattering caused by the perpendicular field component. ${ }^{15,32}$ As a result there is a large drop in resistance once the system becomes spin polarized which can be seen at around $13 \mathrm{~T}$. This effect is much larger than seen in larger gap materials such as GaAs (Refs. 5 and 6) and silicon,, where spin-orbit scattering is much weaker. Interestingly this point also corresponds to a noticeable additional peak in the Hall resistance. The magnetic field position of the polarization transition allows us to calculate the spin splitting by writing $g^{*} \mu_{B} B=2 E_{F}$ which gives $g^{*} \mu_{B} B=51.5 \mathrm{meV}$ and $g^{*}$ $=68.6$ (also plotted in Fig. 4), assuming that this has occurred by $13 \mathrm{~T}$. This requires an additional enhancement of the splitting by $\sim 30 \mathrm{meV}$ above the single-particle value. Studies at temperatures up to $30 \mathrm{~K}$ show the magnetoresistance drop decreasing but show no significant change in the field necessary for full polarization.

We now discuss the origin of this giant enhancement of the Zeeman splitting. All three measurement techniques are in agreement that the fully spin-polarized system has g-factors of order 40-70, representing a more than $100 \%$ increase over the single-particle values and measured at magnetic fields often in excess of $10 \mathrm{~T}$. This requires an additional contribution to the spin splitting of order $10-30 \mathrm{meV}$. The evidence also clearly requires that this enhancement should be dependent upon the spin polarization of the system, thus suggesting a many-body effect. The low effective mass and high dielectric constant of InSb mean that the measurement regime studied above is one in which the average interparticle spacing, as measured by the parameter $\mathrm{r}_{s}=a_{B} / \sqrt{\pi n}$, is of order $0.15-0.3$ where Coulomb effects are expected to be small. ${ }^{1-3}$ This is consistent with the fact that the g-factors measured for the unpolarized system are close to the single-particle values. Calculations ${ }^{1,3,33}$ suggest that the polarization dependence of the spin susceptibility, which can in principle be measured by the tilted field method, ${ }^{1}$ will only give a contribution to the spin splitting of order $2 \mathrm{meV}$ for the InSb system, but detailed calculations are not reported for such small values of $r_{s}$. Experimentally the work on larger mass systems such as GaAs, ${ }^{5,6,22,34}$ silicon,,${ }^{9,10}$ and $\mathrm{CdTe},{ }^{8,35}$ which all have much smaller single-particle g-factors, find large proportional increases in the spin susceptibility at larger values of $r_{s}$ but the total Coulomb contribution to the splittings measured can be estimated to be still only in the region of 1-3 meV.

Spin-orbit (Rashba) coupling contributions to the singleparticle splitting ${ }^{12}$ are also of a similar magnitude. These have been studied optically by Khodaparast et al. ${ }^{36}$ in very similar samples to those used here and found to be only of order $1 \mathrm{meV}$ at low fields in good agreement with theory ${ }^{13}$ which predicts that they become smaller at higher fields. Electron-nuclear-spin interactions, as discussed by Overhauser, ${ }^{37}$ are also much too small to significantly increase the splitting. Theory suggests ${ }^{38}$ that even if all of the polarization were transferred to the nuclei, this could only contribute $\sim 0.4 \mathrm{meV}$ to the splitting at $13 \mathrm{~T}$, while experiments on bulk InSb have reported values $\sim 100 \times$ smaller $^{39}$ than this. The absence of any temperature dependence between $25 \mathrm{mK}$ and $1.5 \mathrm{~K}$ in the coincidence measurements, and up to $30 \mathrm{~K}$ in the depolarization transition measurements also suggest that electron-nuclear-spin interactions are not playing a role.

None of the existing theories seem therefore to provide an explanation of the behavior which we observe. We note that a significant difference between the samples studied here and those studied previously are the much larger single-particle splittings caused by the narrow-gap band structure, which mean that most data is taken in the regime where the singleparticle splittings are of order 7-28 meV (total B field 4-16 T). These values are significantly larger than the homogeneous broadening of the Landau levels as deduced from either the mobilities $(\sim 0.5 \mathrm{meV})$ or cyclotron resonance linewidths $\sim 0.7 \mathrm{meV}$ (Ref. 27) and usually exceed the inhomogeneous broadening of $\sim 8 \mathrm{meV}$, as deduced from a Dingle analysis of the Shubnikov-deHaas oscillations studied here. ${ }^{40}$ This suggests that under most circumstances total separation of all spin states is achieved, rather than only that of the delocalized states needed to form strongly resolved resistivity minima. ${ }^{31}$ By contrast many of the measurements on systems such as GaAs are made under conditions where the single-particle splittings are considerably smaller than the broadening. ${ }^{7,22,34,41}$ As a result the observation of spin split peaks is found to be critically dependent on the broadening and is less dependent on the magnitude of the single-particle splittings. ${ }^{34,41}$ We speculate therefore that the large initial single-particle splittings and even larger total values including enhancement, may lead to significantly increased interaction effects due to the relatively smaller importance of disorder effects. A further possibility which arises due to the narrow gap band structure is that the Rashba coupling may be interacting with the exchange effects to enhance one or other of the two terms. 
In conclusion therefore we have demonstrated that quantum wells based on the narrow-band-gap material InSb can demonstrate remarkably large values for the Zeeman splitting when the electron system becomes fully polarized. This supports recent suggestions ${ }^{1,3}$ that the spin susceptibility of a two-dimensional electron gas should be polarization dependent but the magnitude of the effect reported here is much larger than expected and much larger than observed recently in larger mass systems. ${ }^{8}$

The authors would like to acknowledge the support provided to QinetiQ by the ES Domain of the UK MoD Research Programme administered through the Research Acquisition Organisation. B.N. acknowledges support from the Ministry of Science and Technology, Thailand.

*r.nicholas@physics.ox.ac.uk

${ }^{1}$ Y. Zhang and S. DasSarma, Phys. Rev. Lett. 96, 196602 (2006).

${ }^{2}$ Y. Zhang and S. DasSarma, Phys. Rev. Lett. 95, 256603 (2005).

${ }^{3}$ C. Attaccalite, S. Moroni, P. Gori-Giorgi, and G. B. Bachelet, Phys. Rev. Lett. 88, 256601 (2002).

${ }^{4}$ E. Tutuc, E. P. De Poortere, S. J. Papadakis, and M. Shayegan, Phys. Rev. Lett. 86, 2858 (2001).

${ }^{5}$ E. Tutuc, S. Melinte, and M. Shayegan, Phys. Rev. Lett. 88, 036805 (2002).

${ }^{6}$ J. Zhu, H. L. Stormer, L. N. Pfeiffer, K. W. Baldwin, and K. W. West, Phys. Rev. Lett. 90, 056805 (2003).

${ }^{7}$ Y.-W. Tan, J. Zhu, H. L. Stormer, L. N. Pfeiffer, K. W. Baldwin, and K. W. West, Phys. Rev. B 73, 045334 (2006).

${ }^{8}$ F. Perez, C. Aku-leh, D. Richards, B. Jusserand, L. C. Smith, D. Wolverson, and G. Karczewski, Phys. Rev. Lett. 99, 026403 (2007).

${ }^{9}$ S. A. Vitkalov, H. Zheng, K. M. Mertes, M. P. Sarachik, and T. M. Klapwijk, Phys. Rev. Lett. 87, 086401 (2001).

${ }^{10}$ A. A. Shashkin, S. V. Kravchenko, V. T. Dolgopolov, and T. M. Klapwijk, Phys. Rev. Lett. 87, 086801 (2001).

${ }^{11}$ T. Ando and Y. Uemura, J. Phys. Soc. Jpn. 37, 1044 (1974).

${ }^{12}$ Y. Bychkov and E. Rashba, J. Phys. C 17, 6039 (1984).

${ }^{13}$ W. Zawadzki and P. Pfeffer, Semicond. Sci. Technol. 19, R1 (2004).

${ }^{14}$ W. Desrat, F. Giazotto, V. Pellegrini, F. Beltram, F. Capotondi, G. Biasiol, L. Sorba, and D. K. Maude, Phys. Rev. B 69, 245324 (2004).

${ }^{15}$ W. Desrat, F. Giazotto, V. Pellegrini, M. Governale, F. Beltram, F. Capotondi, G. Biasiol, and L. Sorba, Phys. Rev. B 71, 153314 (2005).

${ }^{16}$ J. C. Chokomakoua, N. Goel, S. J. Chung, M. B. Santos, J. L. Hicks, M. B. Johnson, and S. Q. Murphy, Phys. Rev. B 69, 235315 (2004).

${ }^{17}$ R. J. Nicholas, M. A. Brummell, J. C. Portal, K. Y. Cheng, and A. Y. Cho, Solid State Commun. 45, 911 (1983).

${ }^{18}$ T. P. Smith III and F. F. Fang, Phys. Rev. B 35, 7729 (1987).

${ }^{19}$ S. Brosig, K. Ensslin, A. G. Jansen, C. Nguyen, B. Brar, M. Thomas, and H. Kroemer, Phys. Rev. B 61, 13045 (2000).

${ }^{20}$ F. Fang and P. Stiles, Phys. Rev. 174, 823 (1968).

${ }^{21}$ R. J. Nicholas, R. J. Haug, K. v. Klitzing, and G. Weimann, Phys. Rev. B 37, 1294 (1988).

${ }^{22}$ D. R. Leadley, R. J. Nicholas, J. J. Harris, and C. T. Foxon, Phys. Rev. B 58, 13036 (1998).

${ }^{23}$ D. Shoenberg, Magnetic Oscillations in Metals (Cambridge University Press, Cambridge, England, 1984).

${ }^{24}$ J. M. S. Orr, A. M. Gilbertson, M. Fearn, O. W. Croad, C. J. Storey, L. Buckle, M. T. Emeny, P. D. Buckle, and T. Ashley, Phys. Rev. B 77, 165334 (2008).

${ }^{25}$ E. P. De Poortere, E. Tutuc, S. Papadakis, and M. Shayegan, Science 290, 1546 (2000).

${ }^{26}$ S. Koch, R. J. Haug, K. v. Klitzing, and M. Razeghi, Phys. Rev. B 47, 4048 (1993).

${ }^{27}$ J. M. S. Orr, K.-C. Chuang, R. J. Nicholas, L. Buckle, M. T. Emeny, and P. D. Buckle, Phys. Rev. B 79, 235302 (2009).

${ }^{28}$ G. A. Khodaparast, R. Meyer, X. Zhang, T. Kasturiarachchi, R. E. Doezema, S. J. Chung, N. Goel, M. B. Santos, and Y. J. Wang, Physica E 20, 386 (2004).

${ }^{29}$ C. D. Summers, R. D. Dennis, B. Wherrett, P. G. Harper, and S. D. Smith, Phys. Rev. 170, 755 (1968).

${ }^{30}$ T. Ando and Y. Uemura, J. Phys. Soc. Jpn. 36, 959 (1974).

${ }^{31}$ R. J. Nicholas, R. Stradling, and R. Tidey, Solid State Commun. 23, 341 (1977).

${ }^{32}$ B. Das, S. Datta, and R. Reifenberger, Phys. Rev. B 41, 8278 (1990).

${ }^{33}$ I. L. Aleiner and L. I. Glazman, Phys. Rev. B 52, 11296 (1995).

${ }^{34}$ B. A. Piot, D. K. Maude, M. Henini, Z. R. Wasilewski, K. J. Friedland, R. Hey, K. H. Ploog, A. I. Toropov, R. Airey, and G. Hill, Phys. Rev. B 72, 245325 (2005).

${ }^{35}$ C. Aku-Leh, F. Perez, B. Jusserand, D. Richards, W. Pacuski, P. Kossacki, M. Menant, and G. Karczewski, Phys. Rev. B 76, 155416 (2007).

${ }^{36}$ G. A. Khodaparast, R. E. Doezema, S. J. Chung, K. J. Goldammer, and M. B. Santos, Phys. Rev. B 70, 155322 (2004).

${ }^{37}$ A. W. Overhauser, Phys. Rev. 92, 411 (1953).

${ }^{38}$ J. R. Morton and K. F. Preston, J. Magn. Reson. 30, 577 (1978).

${ }^{39}$ W. Hofmann, G. Denninger, and H. Pascher, Phys. Rev. B 48, 17035 (1993).

${ }^{40}$ Conventional Dingle analysis of the damping of the oscillations is performed at $\sim 70 \mathrm{deg}$ where the two sets of spin states are nearly coincident, thus leading to an unsplit single series of oscillations with no higher harmonic content.

${ }^{41}$ B. A. Piot, D. K. Maude, M. Henini, Z. R. Wasilewski, J. A. Gupta, K. J. Friedland, R. Hey, K. H. Ploog, U. Gennser, A. Cavanna, D. Mailly, R. Airey, and G. Hill, Phys. Rev. B 75, 155332 (2007). 